\title{
A nice stratagem to treat poikiloderma of Civatte, evoked by fragrances and essential oils, by means of a resin that is commonly employed to finish mandolins and Maggiolini's furniture
}

\section{Piotr Brzezinski, Lorenzo Martini}

\author{
University of Siena, Department of Pharmaceutical Biotechnologies, Via A. Moro 2, 53100 Siena, Italy
}

Corresponding author: Prof. Lorenzo Martini, M.Sc., E-mail: martinil163@libero.it

\begin{abstract}
Since the exact ethiology of Poikiloderma of Civatte is hitherto unknown, even if many researchers assert that hormonal factors and photosensitising components of cosmetics and toiletries, especially perfumes, do valuably contribute to its occurrence and moreover as there is no specific medical treatment for this condition. The AA of this paper have ideated a specific gélée apt to create a protective and curative layer onto the damaged skin, to spread the night. It is interesting the fact that the nocturnal usage of this gélée permits to the patient to wear whichever fragrance the day after with no risk of occurrence of relapse of the poikiloderma itself.
\end{abstract}

Key words: Poikiloderma of Civatte; shellac, Lycopus extract

\section{INTRODUCTION}

Poikiloderma of Civatte is a common, benign skin condition that mainly affects the skin on cheeks and on the sides of the neck. It mainly affects women, even if men who profusely wear after shaving eaux de toilette or eaux de parfum, especially made of Lavender or Bergamot, are not exempted from the occurrence of this disease [1].

It characteristically spares the shaded area under the chin. The skin in the affected area is red-brown with prominent hair follicles. The term "poikiloderma" refers to a change in the skin where there is thinning, increased pigmentation and dilation of the fine blood vessels (telangiectasia).

Civatte was a French dermatologist who in 1923 first described a common weathering change that affects the skin of the sides and front of the neck.
The exact ethiology of this syndrome is hitherto unknown, even if many researchers assert that:

a) Hormonal factors

b) Photosensitising components of cosmetics and toiletries, especially perfumes may valuably contribute to its occurrence.

There is no specific medical treatment for this condition. The patient should be educated about avoiding sun exposure and the correct use of sunscreens, but even avoiding use and/or abuse of fragrances and cosmetic items that contain those exuberantly.

Preventive strategies shall be: Sun protection including daily broad spectrum SPF; Avoiding all perfumes on or near the affected area, including those in soap; Hydroquinone-containing preparations that may help fade the pigmentation; Exfoliants including long term use of alpha hydroxy-acids and/ or tretinoin.

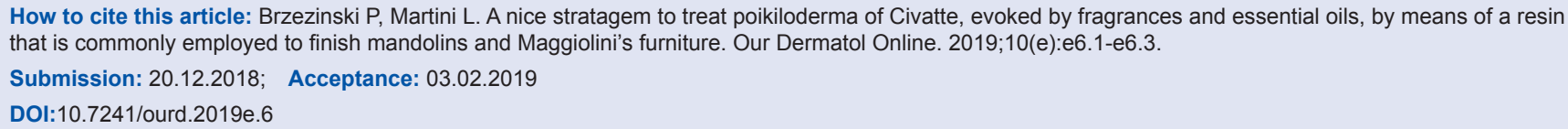


Pulsed dye laser (PDL) and intense pulsed light (IPL) treatments seem the best way to reduce the telangiectasia and pigmentation.

Fragrances are important sources of ACD. Fragrances are found in many cosmetics, as well as more traditionally in perfume or cologne form. Fragrances, including fragrance mix, balsam of Peru, and cinnamic aldehyde are the most commonly identified allergens in cosmetic-induced ACD $[2,3]$.

It is disconcerting to notice that when a cosmetic product lists "essential oils" as an ingredient (according to EC rule 1223/2009) consumers may not recognize this as fragrance objectively.

In order to standardize a regular allergy test, valid through all the world, three types of combinés of synthetic or natural fragrances have been ideated and these are, respectively [4-8]:

Mix 1, that contains:

Cinnamal* 1.0; Cinnamyl alcohol* 2.0; Amylcinnamal* 2.0; Eugenol* 2.0; Isoeugenol* 2.0; Geraniol* 2.0; Evernia prunastri absolute* 2.0; Hydroxycitronellal* 2.0; Narcissus absolute 2.0; Musk xylene 1.0; Methyl anthranilate 5.0; Musk moskene 1.0; Musk ketone 1.0; Jasmine synthetic 2.0; Benzyl salicylate 10.0; Benzyl alcohol 10.00; Vanillin 10.00; Lavender absolute 2.0; Propolis 10.0; Myroxylon pereirae 25.0;

Mix II, that contains:

2H-1-Benzopyran-2-one; Coumarin; Tonka bean camphor; 1,2-Benzopyrone; 2-Oxo-1,2-benzopyran; 2-Propenoic acid, 3-(2-hydroxyphenyl)-, deltalactone; 2-Propenoic acid, 3-(2-hydroxyphenyl)delta-lactone; 2H-1-Benzopyran-2-one; 3-(2-Hydroxyphenyl)-2-propenoic deltalactone; 5,6-Benzo-alpha-pyrone; Benzo-alphapyrone; Cinnamic acid, o-hydroxy-, deltalactone; Coumarinic anhydride; Coumarinic lactone; cis-o-Coumaric acid anhydride; ciso-Coumarinic acid lactone; o-Coumaric acid lactone; o-Hydroxycinnamic acid lactone; o-Hydroxycinnamic lactone; 4-(4-Hydroxy-4methylpentyl)-3-cyclohexene-1-carboxaldehyde; 4-(4-Hydroxy-4-methylpentyl)cyclohex-3enecarbaldehyde; 2,6-Dimethyl-2-octen-8-ol; 3,7-Dimethyl-6-octen-1-ol; Cephrol; Citronellol (ex. Java citronella oil); Citronellol (natural); Elenol; Rhodinol; 3,7,11-Trimethyl-2,6,10-dodecatrienol;
3,7,11-Trimethyl-2,6,10-dodecatrien-1-ol; Trimethyl dodecatrienol

And Mix III:

hydroxyisohexyl 3-cyclohexene carboxaldehyde

Allergens are to be applied to the upper back for 2 days, and the results are recorded on day (D)2 and D3, according to international recommendations $[9,10]$.

In this paper the co-author, who has been always using to spread pure essential oils onto his cheeks and neck since decades, presents an harsh poikiloderma and the corresponding author represents the dermatologist who has treated his skin affection, even if the two AA had ideated the formula of the cosmetic item apt to clear reddish and purple hair follicles and protect to act positively on telangiectasias.

The combinaison of cosmetic ingredients of our formula includes the following ingredients: Fumed Silica; Grapeseed oil; Shellac (CAS 9000-59-3).

Lycopus coreanus leaf extract, that manifests exceptional vasoconstrictor properties towards capillaries in telengiactasias and chloasmas.

Shellac is a resin secreted by the female lac bug, that lives on trees in the forests of Bangladesh and Thailand. When the lac (or resin) is dissolved in ethanol, it is useful for food glaze and wood finish especially for the production of mandolins, ukuleles and Maggiolini's furniture. Shellac functions as a tough natural primer, sanding sealant, tannin-blocker, odourblocker, stain, and high-gloss varnish, possesses good insulation qualities and it seals out moisture.

Instead of dissolving the resin in alcohol, the AA disperded it in a gélée made of silica and vegetable oil at high temperature so that shellac may disclose all its barrier functions onto injured skin.

Oil acts as a magnificent emollient and Lycopus extract, as Urtica dioica, let vessels and capillaries responsible of telengiactasias and chloasmas to grow pale and stop to evoke itch and unpleasant redness, owing to its powerful vasoconstrictor and hemostatic activities.

\section{MATERIALS AND METHODS}

The co-author of this paper was suffering from a poikiloderma of Civatte and had tried manifold 
remedies, natural as well and moreover steroid pomades or jellies, with no satisfactory visible results.

He made up his mind to attempt to cure the disease by means of this special cosmetic salbe and used to spread over the cheeks and the sides of his neck every night before to sleep for two entire weeks.

\section{RESULTS}

It is suggestive to remark that during the first week the hair follicles tended to deflate and soften progressively.

During the second week, redness and itch disappear at all in all the interested areas of face and neck.

We suppose this phenomenon is due to the fact that Lycopus extract acts very slowly as vasoconstrictor.

\section{CONCLUSIONS}

It is interesting to highlight the fact that the coauthor could wear all types of pure fragrances or mix of perfumes or eaux de toilette every morning, after having washed and rinsed his face and neck.

During the day successive to the application of the gélée, no symptoms or visible manifestations of the poikiloderma were recognizable at all.

\section{Statement of Human and Animal Rights}

All procedures followed were in accordance with the ethical standards of the responsible committee on human experimentation (institutional and national) and with the Helsinki Declaration of 1975, as revised in 2008.

\section{Statement of Informed Consent}

Informed consent was obtained from all patients for being included in the study.

\section{REFERENCES}

1. Khunkhet S, Wattanakrai P. The possible role of contact sensitization to fragrances and preservatives in poikiloderma of Civatte. Case Rep Dermatol. 2014;6:258-63.

2. Ahmad W, Latif I, Sheikh G, Hassan I, Rasool F, Keen A. Patch testing - Revisited. Our Dermatol Online. 2017;8:224-8.

3. Parajuli S, Paudel V, Paudel U, Pokhrel DB. Pattern of patch test reactivity among patients with clinical diagnosis of contact dermatitis: A hospital-based study. Our Dermatol Online. 2017;8:389-92.

4. Jing L, Li LF. Contact sensitization to fragrances other than fragrance mix I in China. Contact Dermatitis. 2015;73:248-58.

5. Martini L, Valle A. Cross-linked natural gum resins, when inserted in shampooing product, result infallible to eliminate several metallic ions risky for hair keratin. Our Dermatol Online. 2016;7:160-4.

6. Mann J, McFadden J P, White J M, White I R, Banerjee P. Baseline series fragrance markers fail to predict contact allergy. Contact Dermatitis. 2014:70:276-81.

7. Martini L. The rediscovery of the Redwood orpiment and a cocktail of plants macerates containing arbutin to defeat the ArribasSilvestre's syndrome in a bien agée upper class lady. Our Dermatol Online. 2017;8:399-401.

8. Gupta M. Pigmented contact dermatitis to p-paraphenylenediamine in a textile factory worker. Our Dermatol Online. 2017;8:424-6.

9. Sharma VK, Bhatia R, Yadav CP. Clinical Profile and Allergens in Pigmented Cosmetic Dermatitis and Allergic Contact Dermatitis to Cosmetics in India. Dermatitis. 2018;29:264-9.

10. Brambarova DA, Yankova RK. Skin sensitivity - extremely positive reaction for nickelsulfate hexahydrate in association with occupational allergens. Our Dermatol Online. 2017;8:349-51.

Copyright by Piotr Brzezinski, et al. This is an open access article distributed under the terms of the Creative Commons Attribution License, which permits unrestricted use, distribution, and reproduction in any medium, provided the original author and source are credited.

Source of Support: Nil, Conflict of Interest: None declared. 